\title{
Treatment of Temporomandibular Disorders of Muscular Origin with a Silicon Oral Device (Alifix®): Electromyographic Analysis
}

\author{
Marcello Maddalone ${ }^{1}$, Edoardo Bianco ${ }^{2}$, Alessandro Nanussi ${ }^{3}$, Giulia Costa ${ }^{4}$, Marco Baldoni ${ }^{5}$
}

\begin{abstract}
Aim: The aim of this work was to evaluate if the use of a silicone device for muscular rebalancing (Alifix ${ }^{\circledR}$ ) can be useful in treating of temporomandibular disorders (TMD) of muscular origin and improving the electromyographic indexes of the chewing muscles.

Materials and methods: Thirteen patients ( $11 \mathrm{~F}$ and $2 \mathrm{M}$ aged between 24 years and 65 years) with TMD of muscular origin according to diagnostic criteria (DC)/TMD were involved. At the first visit (T0), each patient reported the pain intensity of masseters and temporal muscles. A surface electromyography (EMG) was performed using Teethan ${ }^{\circledast}$ (Teethan S.p.A.) and then Alifix ${ }^{\circledast}$ was delivered instructing the patient on its use. Each subject was visited again after 1 month (T1) and 2 months (T2). New EMG had been made at T1 and T2, and patients were asked again to report the pain intensity. Statistical analysis was calculated between T0 and T1,T1 and T2, and T0 and T2 for all EMG, and muscle pain measurements by Wilcoxon test with statistical significance $p<0.05$.

Results: Regarding the pain values between $\mathrm{T} 0$ and $\mathrm{T} 1, \mathrm{~T} 1$ and $\mathrm{T} 2$, and $\mathrm{T} 0$ and $\mathrm{T} 2$, the difference is statistically significant, since the intensity of pain between T0 and T2 is decreased, if not disappeared, in $90 \%$ of cases. The use of Alifix ${ }^{\oplus}$ also determined a gradual improvement in the values of the EMG indexes, which, however, is not statistically significant.

Conclusion: The effectiveness of Alifix ${ }^{\circledast}$ is demonstrated clinically but not at an instrumental level. Further studies involving a larger sample and taking longer therapy duration are needed.

Clinical significance: Alifix ${ }^{\oplus}$ works by improving the blood circulation of the muscle, which allows the removal of catabolites with a consequent reduction of the algic symptomatology and promotes a greater supply of oxygen. It also encourages a conversion of IIA type muscle fibers into slow-twitch type I fibers that are more resistant to neuromuscular fatigue.

Keywords: Alifix, Electromyography, Muscle pain, Oral osteopathy, Temporomandibular disorders.

The Journal of Contemporary Dental Practice (2019): 10.5005/jp-journals-10024-2704
\end{abstract}

\section{A IM}

Temporomandibular disorders are among the most difficult pain pathologies to treat, are a complex disease, and their nature has not yet been fully understood. Their origin is often uncertain, even after the execution of all clinical and instrumental diagnostic procedures. $^{1-6}$

A large percentage of patients with TMD report pain on the masticatory muscles and have signs and symptoms of muscle alteration. ${ }^{2-8}$

Among the different devices proposed for the resolution of TMD, oral splints have proved effective in reducing pain, but there are other devices that allow a controlled training of chewing muscles. ${ }^{2,3,5,9}$

Pain control as in other dental disciplines can be achieved and controlled with different devices yet described or innovative. ${ }^{10-21}$

Several studies state that the masticatory musculature of symptomatic patients with temporomandibular disorders (TMD) produces reduced electrical potentials and a less efficient clenching force. Hence, the muscles become more easily subjected to neuromuscular fatigue when compared to the force developed by healthy subjects. . $^{3,6,8,8,22}$

In these situations, adequate muscle training determines a better function, while reducing pain and disability. Changes in muscle fiber are also promoted, both in terms of size and composition. With this training, strength and resistance to neuromuscular fatigue also increase. ${ }^{23,24}$
${ }^{1}$ Department of Orthodontics, San Gerardo Hospital, University of Milano Bicocca, Monza, Milano Bicocca, Italy

${ }^{2-5}$ Department of Surgery and Translational Medicine, San Gerardo Hospital, University of Milano Bicocca, Monza, Milano Bicocca, Italy

Corresponding Author: Edoardo Bianco, Department of Surgery and Translational Medicine, San Gerardo Hospital, University of Milano Bicocca, Monza, Milano Bicocca, Italy, Phone: +39 0392333485, e-mail: edoardo.bianco13@gmail.com

How to cite this article: Maddalone M, Bianco E, Nanussi $A$, et al. Treatment of Temporomandibular Disorders of Muscular Origin with a Silicon Oral Device (Alifix ${ }^{\circledast}$ ): Electromyographic Analysis. J Contemp Dent Pract 2019;20(12):1367-1374.

Source of support: Nil

Conflict of interest: None

The training of the masticatory muscles has already been successfully tested in healthy subjects. In particular, the protocols that provided exercises based on mastication (isotonic contraction) have shown to have a greater compliance compared to protocols based on exercises based on clenching (isometric contraction). ${ }^{3,23-25}$

In this study, subjects with TMD of muscular origin have been proposed to use a device (Alifix ${ }^{\oplus}$ ) designed for the training of chewing muscles to verify the effects of muscular training on the surface EMG indexes chewing muscles and the intensity of pain.

(c) The Author(s). 2019 Open Access This article is distributed under the terms of the Creative Commons Attribution 4.0 International License (https://creativecommons. org/licenses/by-nc/4.0/), which permits unrestricted use, distribution, and non-commercial reproduction in any medium, provided you give appropriate credit to the original author(s) and the source, provide a link to the Creative Commons license, and indicate if changes were made. The Creative Commons Public Domain Dedication waiver (http://creativecommons.org/publicdomain/zero/1.0/) applies to the data made available in this article, unless otherwise stated. 
Alifix ${ }^{\circledast}$ is made of silicone and consists of two separate elements, right and left, mirrored to each other (Figs 1 and 2).

Each element is formed by a vertical disk that rests on the cheek and a horizontal part with a wedge to be inserted between the teeth, whose thickness varies from one end to the other.

Once positioned between the teeth Alifix ${ }^{\oplus}$ uses chewing and swallowing movement to perform muscle training exercises, in order to stimulate and rebalance the muscular system of the mouth.

\section{Materials and Methods}

For this study, 13 patients ( 11 female and 2 male between the age of 24 years and 65 years) with TMD of muscular origin were recruited, and whose diagnosis was placed after anamnesis and gnathologic visit conducted following the DC/TMD (diagnostic criteria of temporomandibular disorders of 2014). ${ }^{26}$

This study was conducted at the Clinic of Dentistry, Department of Surgery and Translational Medicine of San Gerardo Hospital, in Monza. Each patient subscribed the informed consent at the beginning of the treatment.

The inclusion criteria were permanent dentition with premolar contacts and at least one molar contact for hemiarcata; they should not suffer from acute problems (inflammatory or infectious); periodontal disease in the acute phase and mobility of the teeth; mono- or bilateral acute joint locking; cognitive/neurological deficits and nervous pathologies; tumors; and current or previous traumas in the head-neck area (trauma/mandibular or dental fractures, tissue lesions). Further exclusion criteria were active orthodontic therapy at the time of screening and use of analgesics, anti-inflammatories, and drugs for the treatment of psychiatric illnesses.

For the evaluation of pain, the research diagnostic criteria (RDC)/TMD questionnaires of 2011 have been used, because based on a numerical scale, more useful for statistical analysis, unlike those belonging to DC/TMD that detect pain solely on its presence or absence, without attributing a number to describe its intensity.

At each appointment, the patient reported a value between 0 and 3 to describe the pain intensity of masseters and temporal muscles. The averages of these values are shown in Table 1.

Patients with TMD of muscular origin were recruited for the study and the first visit corresponds to the TO phase.

During the first visit, the patients were subjected to surface EMG of masseters and temporal muscles using Teethan ${ }^{\circledast}$ (Teethan S.p.A.).

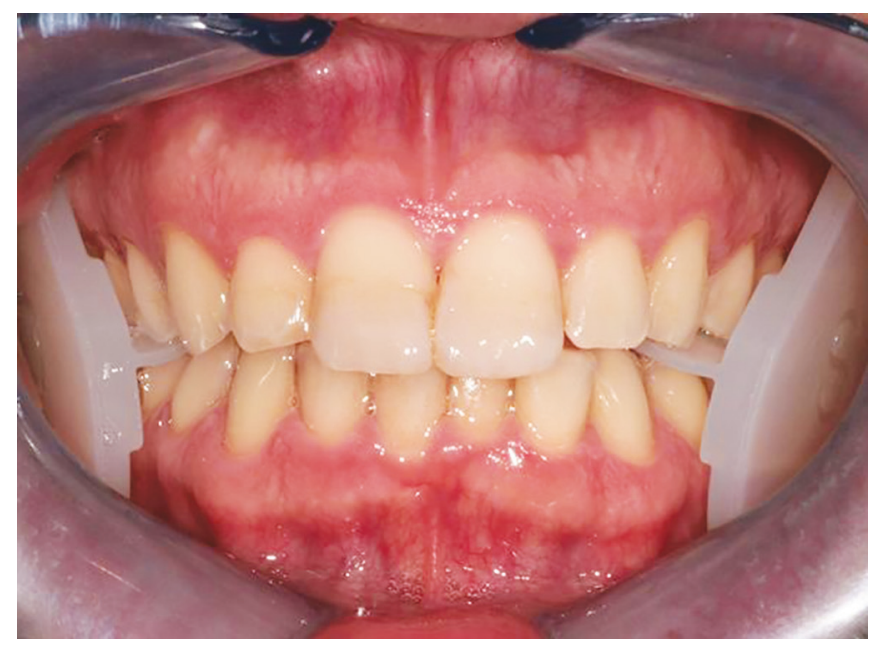

Fig. 1: Correct positioning of Alifix ${ }^{\circledast}$ in the mouth
The electrodes used were bipolar circular-shaped with a diameter of $1 \mathrm{~cm}$ and pre-gelled with saline base conductive gel. After cleansing the skin with gauze soaked in $90 \%$ denatured ethyl alcohol to remove the sebum, they were placed at the belly of the masseters (at the intersection of the tragus-commissure labial and exocanthion-gonion lines) and along the anterior border of the temporal muscle, at the level of the coronal suture, after palpation of the muscles in maximum clenching. The inter-electrode distance was kept constant at $2 \mathrm{~cm}$ (Fig. 3).

The acquisition protocol used consists of two tests with a duration of 5 seconds each:

- Clenching test on cottons (to be placed on the 5th/6th element, without constraining the canine guidance and not too close to the cheeks not to alter proprioception)

- Test in maximum voluntary clenching (MVC) on teeth.

The test on cotton rolls allowed to calibrate the EMG on the specific patient analyzed. If the calibration were not performed, the investigations would have been influenced by several factors such as cross-talk (analysis of nearby muscles), skin conductivity (variable depending on the tissue composition) and skin condition (sweating), and position of the probes. What was analyzed during the calibration is not the occlusion, but the maximum muscle activity expressed as the electrical activity that the muscles can express without the dental contact (ideal condition).

This value is used to normalize the data, term which means that the software automatically compares the calibration and acquisition tests and evaluates, through EMG indexes, how the neuromuscular activity changes once the dental contact is inserted.

This working protocol allows obtaining repeatable results and reliable normal ranges. ${ }^{27}$

Each patient underwent two recordings: a test on cottons with eyes open and a test in MVC on teeth with eyes open.

During the test, the patient was seated on a straight back chair without metal structures, with the feet touching the floor, the hands along the thighs, and the head straight with the eyes facing a plain wall. During the 5 seconds of each trial, the patient was continuously encouraged to keep the maximum clenching strength.

Measurements taken using EMG were processed by Teethan ${ }^{\circledast}$ software which returns numerical values for each EMG index analyzed: percentage of overlapping coefficient of temporal muscles and masseters (POC TA, POC MM), barycenter, the center

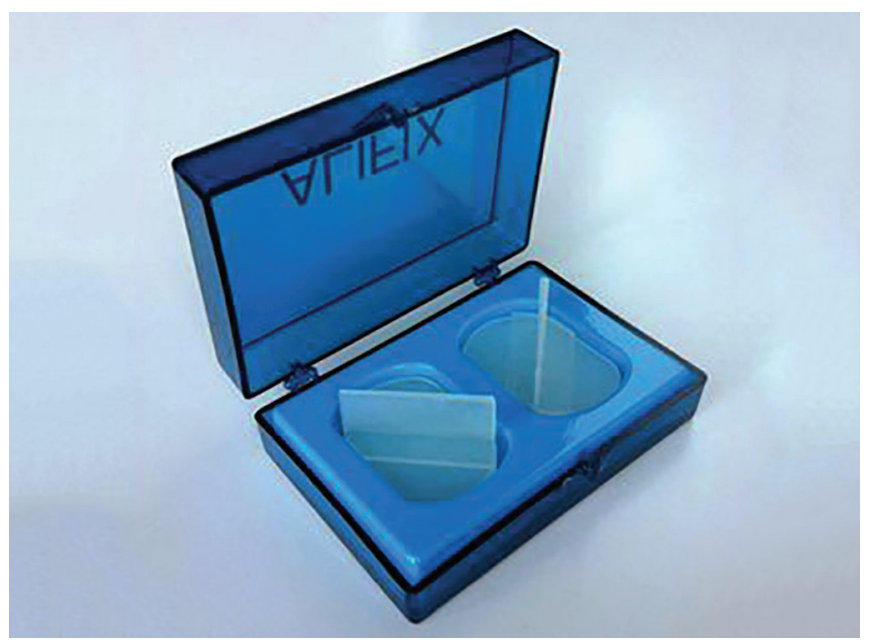

Fig. 2: Alifix ${ }^{\circledast}$ device 
Table 1: Mean pain of temporal muscles, masseters, and both from T0 to T2

\begin{tabular}{|c|c|c|c|c|c|c|c|}
\hline \multirow[b]{2}{*}{ Patient } & \multicolumn{4}{|c|}{ Muscles } & \multirow[b]{2}{*}{ Total mean pain } & \multirow{2}{*}{$\begin{array}{l}\text { Temporals mean } \\
\text { pain }\end{array}$} & \multirow{2}{*}{$\begin{array}{l}\text { Masseters mean } \\
\text { pain }\end{array}$} \\
\hline & $R T$ & $L T$ & $R M$ & $L M$ & & & \\
\hline \multicolumn{8}{|l|}{ E.D. } \\
\hline TO & 1 & 2 & 1 & 1 & 1.25 & 1.5 & 1 \\
\hline $\mathrm{T} 1$ & 2 & 1 & 2 & 2 & 1.75 & 1.5 & 2 \\
\hline $\mathrm{T} 2$ & 1 & 3 & 0 & 0 & 1 & 2 & 0 \\
\hline \multicolumn{8}{|l|}{ G.G. } \\
\hline T0 & 2 & 0 & 2 & 3 & 1.75 & 1 & 2.5 \\
\hline $\mathrm{T} 1$ & 0 & 2 & 0 & 2 & 1 & 1 & 1 \\
\hline $\mathrm{T} 2$ & 0 & 0 & 0 & 0 & 0 & 0 & 0 \\
\hline \multicolumn{8}{|l|}{ R.G. } \\
\hline T0 & 3 & 3 & 1 & 1 & 2 & 3 & 1 \\
\hline $\mathrm{T} 1$ & 3 & 2 & 1 & 1 & 1.75 & 2.5 & 1 \\
\hline $\mathrm{T} 2$ & 1 & 1 & 0 & 0 & 0.5 & 1 & 0 \\
\hline \multicolumn{8}{|l|}{ C.G. } \\
\hline TO & 2 & 0 & 3 & 0 & 1.25 & 1 & 1.5 \\
\hline $\mathrm{T} 1$ & 2 & 1 & 1 & 0 & 1 & 1.5 & 0.5 \\
\hline $\mathrm{T} 2$ & 0 & 0 & 0 & 0 & 0 & 0 & 0 \\
\hline \multicolumn{8}{|l|}{ F.V. } \\
\hline T0 & 3 & 0 & 3 & 0 & 1.5 & 1.5 & 1.5 \\
\hline $\mathrm{T} 1$ & 1 & 0 & 2 & 0 & 0.75 & 0.5 & 1 \\
\hline $\mathrm{T} 2$ & 1 & 0 & 0 & 0 & 0.25 & 0.5 & 0 \\
\hline \multicolumn{8}{|l|}{ B.G. } \\
\hline T0 & 0 & 1 & 0 & 3 & 1 & 0.5 & 1.5 \\
\hline $\mathrm{T} 1$ & 0 & 2 & 0 & 2 & 1 & 1 & 1 \\
\hline $\mathrm{T} 2$ & 0 & 2 & 0 & 2 & 1 & 1 & 1 \\
\hline \multicolumn{8}{|l|}{ R.R. } \\
\hline T0 & 3 & 1 & 0 & 0 & 1 & 2 & 0 \\
\hline $\mathrm{T} 1$ & 0 & 0 & 0 & 0 & 0 & 0 & 0 \\
\hline $\mathrm{T} 2$ & 0 & 0 & 0 & 0 & 0 & 0 & 0 \\
\hline \multicolumn{8}{|l|}{ S.Y. } \\
\hline T0 & 0 & 0 & 3 & 3 & 1.5 & 0 & 3 \\
\hline $\mathrm{T} 1$ & 0 & 0 & 3 & 2 & 1.25 & 0 & 2.5 \\
\hline $\mathrm{T} 2$ & 0 & 0 & 1 & 1 & 0.5 & 0 & 1 \\
\hline \multicolumn{8}{|l|}{ J.J. } \\
\hline T0 & 3 & 2 & 1 & 1 & 1.75 & 2.5 & 1 \\
\hline $\mathrm{T} 1$ & 1 & 1 & 0 & 0 & 0.5 & 1 & 0 \\
\hline $\mathrm{T} 2$ & 0 & 0 & 0 & 0 & 0 & 0 & 0 \\
\hline \multicolumn{8}{|l|}{ M.C. } \\
\hline T0 & 0 & 0 & 2 & 1 & 0.75 & 0 & 1.5 \\
\hline $\mathrm{T} 1$ & 0 & 0 & 0 & 0 & 0 & 0 & 0 \\
\hline $\mathrm{T} 2$ & 0 & 0 & 0 & 0 & 0 & 0 & 0 \\
\hline
\end{tabular}

$\mathrm{RT}$, right temporal muscle; LT, left temporal muscle; RM, right masseter; and LM, left masseter

of muscular work which can be anterior or posterior (BAR), right or left torsion (TORS).

At the end of the first session, Alifix ${ }^{\circledR}$ was delivered to each subject after checking the comfortable position of reference, i.e., with the greater thickness of the wedge placed anteriorly or posteriorly.

Three patients who received Alifix ${ }^{\oplus}$ did not show up at the controls; hence, only 10 patients were considered in the series.

Then the fatigue test was performed: the patient was asked to chew Alifix ${ }^{\circledast}$ to identify the time necessary for the occurrence of the first symptoms of muscular fatigue on masseters or temporal muscles. The time taken to achieve this feeling of muscular fatigue was recorded by the operator using a stopwatch and was used, taking as a reference the training table provided by the manufacturer, to program the exercises that the patient would later perform at home using Alifix ${ }^{\oplus}$. The exercise protocol consisted of an alternation of minutes of chewing Alifix to minutes of rest in which the patient does not chew but keeps Alifix ${ }^{\circledast}$ in the mouth, which however performs its function during swallowing. The goal was to reach 5 minutes of consecutive chewing without feeling 
any muscular fatigue. So, according to the result of the fatigue test, patients were instructed to train with Alifix ${ }^{\circledR}$ once a day increasing the chewing time of 1 minute per week, until reaching the aforementioned 5 minutes.

Each subject in the test group was visited in two other sessions, 1 month (T1) and 2 months (T2) after T0, during which he was again subjected to clinical pain assessment, using the RDC/TMD forms, and EMG of temporal muscles and masseter muscles using Teethan ${ }^{\circledast}$. The patient was then asked to continue therapy with Alifix for up to a month after the symptoms disappeared.

The statistical analysis of the EMG indexes was performed by comparing the values obtained between T0 and T1, T1 and T2, and $\mathrm{T} 0$ and $\mathrm{T} 2$.

\section{Results}

For the evaluation of pain change, a statistical analysis was carried out. The general muscular pain values and that of masseters and temporal muscles alone have been compared between $\mathrm{T} 0$ and $\mathrm{T} 1$, $\mathrm{T} 1$ and $\mathrm{T} 2$, and $\mathrm{T} 0$ and $\mathrm{T} 2$.

Since the data collected do not follow a Gaussian distribution, the Wilcoxon rank test was chosen for all the statistical analyses. Statistical significance was set with $p<0.05$.

Data for statistical analyses were processed in an Excel spreadsheet.

Three patients, who received Alifix ${ }^{\circledR}$ but did not show up at the $\mathrm{T} 1$ or $\mathrm{T} 2$, were not included in the statistical analysis that was made only on 10 patients.

\section{EMG Indexes Variation}

The use of Alifix ${ }^{\circledast}$ has led to a progressive increase in the values of the POC TA, POC MM, and BAR indexes, which however is not statistically significant. The values are shown in Table 2. A single exception is represented by the TORS index which at first decreases and then increases.

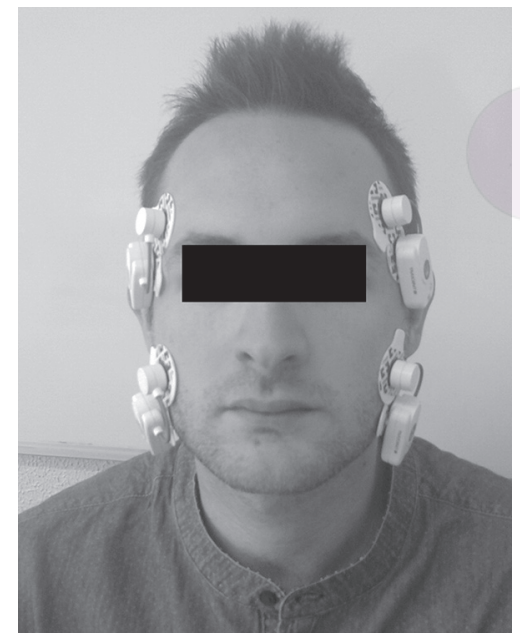

Fig. 3: Electromyographic analysis
However, it is noted that the averages of the POC TA and POC MM indexes go from nonphysiological values, respectively, 74.853 and 74.981 in phase T0 to values that in phase T2 are within the normal range (between 83 and 100); in fact these averages reach the values of 83.377 for POC TA and 86.861 for POC MM. This consideration translates clinically into a more symmetrical distribution of muscular activity of masseters and temporal muscles.

The EMG index BAR of the test group in phase T0 has an average value equal to 79.993 , which increases up to 85.052 in phase $T 2$ after the use of Alifix ${ }^{\oplus}$, reaching the normal range which is between 85 and 115.

The mean TORS index of the test group corresponds to 87.265 in T0 phase and reaches the value of 89.772 after 2 months of therapy with Alifix ${ }^{\circledast}$, falling almost within the normal range that for TORS is between 90 and 100 (Fig. 4).

\section{Pain Variation}

The mean values of muscle pain referred by the patient are reported in Table 1 and the statistical analysis is based on them. In the comparison between $\mathrm{T} 0$ and $\mathrm{T} 1, \mathrm{~T} 1$ and $\mathrm{T} 2$, and $\mathrm{T} 0$ and $\mathrm{T} 2$, the difference between the numerical values relative to the pain scale (i.e., the decrease of the algic symptomatology over time) is statistically significant.

In $80 \%$ of the cases, the test group declared at the second session (T1) a decrease in pain symptoms, $10 \%$ reported worsening and the remaining $10 \%$ did not notice any changes.

At the third session (T2), 50\% of patients reported a total disappearance of pain, $40 \%$ reported a further decrease compared to the second session, and the remaining $10 \%$ did not find benefits from using Alifix ${ }^{\circledR}$.

Regarding solely the temporal muscles, we noticed a decrease in pain in $75 \%$ of the cases from the T0 phase to the T2 phase, while for the masseters alone the pain decreased in $100 \%$ of the cases. The progression of the average general muscular pain of the localized pain in temporal muscles and of the pain located at the masseters for each individual patient is illustrated. The averages related to the intensity of pain, both for masseters and temporal muscles together and separately, are progressively decreasing, demonstrating the resolving effect that Alifix ${ }^{\circledR}$ exerts toward the algic symptoms (Figs 5 to 8).

\section{Conclusion}

The inability to gently chew Alifix ${ }^{\circledR}$ for consecutive 5 minutes without experiencing pain or fatigue to the masseter or temporal muscles is an indication of a TMD of muscular origin. This situation indicates the presence of an altered metabolism of the muscle with changes in the response to proprioceptive stimuli and the need for adequate training of the chewing muscles.

The therapy with Alifix ${ }^{\circledast}$ in subjects with TMD of muscular origin aims to achieve a balanced and symmetrical distribution of muscular forces and to resolve the algic symptomatology.

The changes made by Alifix ${ }^{\circledR}$ have been evaluated from both an instrumental and clinical point of view.

Table 2: Mean electromyographic values (percentage of overlapping coefficient, barycenter, and torsion) from T0 to T2

\begin{tabular}{lllll}
\hline $\begin{array}{l}\text { Mean electromyo- } \\
\text { graphic values }\end{array}$ & Temporal muscles POC & Masseters POC & Mean POC & BAR \\
\hline T0 & 74.853 & 74.981 & 74.9185 & 79.993 \\
T1 & 76.256 & 82.567 & 79.3715 & 80.453 \\
T2 & 83.377 & 86.861 & 85.1195 & 85.032 \\
\hline
\end{tabular}



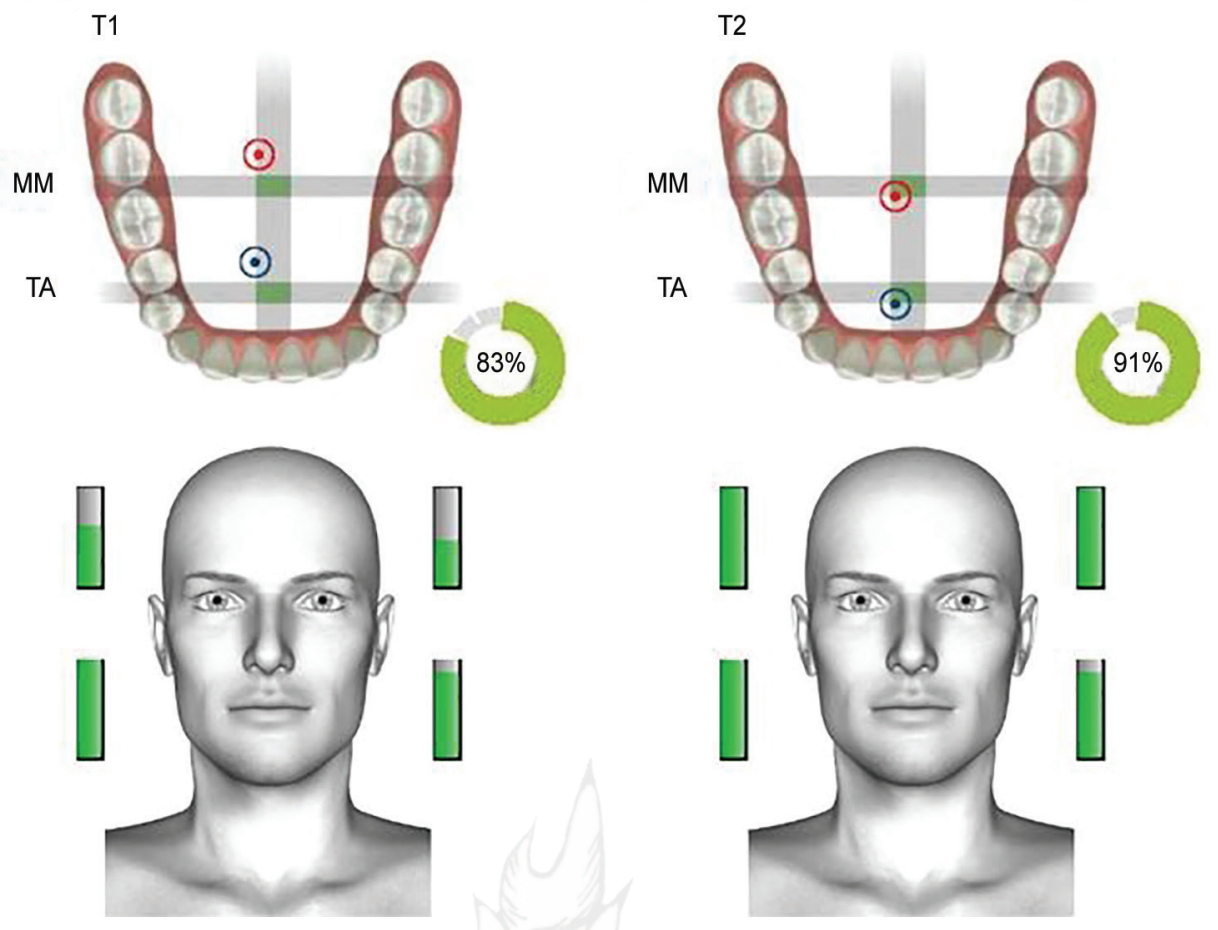

Fig. 4: Example of an electromyographic report: improvements from T1 to T2

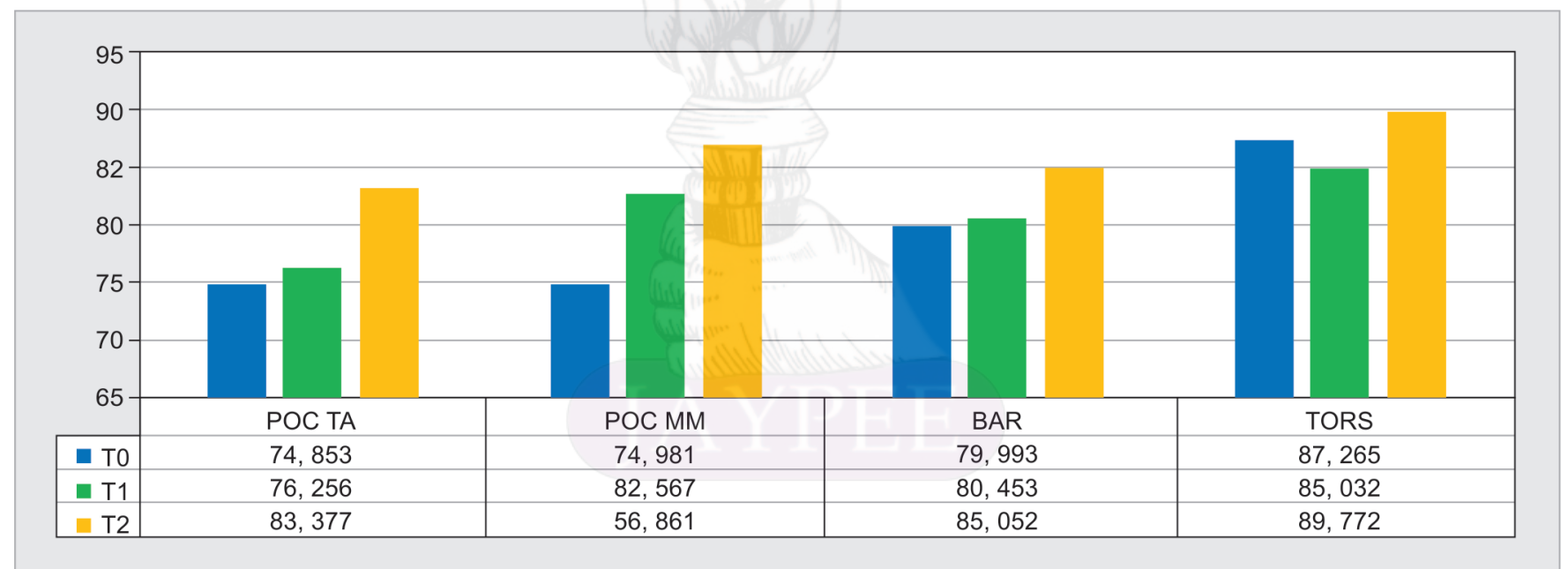

Fig. 5: Mean electromyographic Teethan ${ }^{\circledast}$ (Teethan S.p.A.) indexes. It can be noticed that they increase to higher (i.e., more physiological) values from T0 (before treatment) to T2 (after 2 months of treatment with Alifix ${ }^{\oplus}$ )

Muscle activity plays a dominant role in the physiology of occlusion and on the function and dysfunction of temporomandibular joint (TMJ).

From the instrumental point of view, the EMG is an important aid to assess the state of health of the neuromuscular system and the muscular components of the stomatognathic apparatus, as well as the possible pathological changes of the other components of the system. Occlusal alteration may be due to a disharmonic spatial position of the mandible, resulting in a force that alters the neuromuscular balance of the stomatognathic apparatus and forces the muscles to assume forced and unbalanced positions. When the jaw is moving, there may be dental interference that forces muscle dynamics to disharmonic functional patterns with consequent pathology of the muscles themselves. Alterations in the TMJ can also cause muscular dysfunction that results in painful and dysfunctional syndromes (Fig. 9).

As seen by Pimenta Ferreira et al., patients with TMD have lower muscle oxygen extraction capacity than healthy subjects. ${ }^{28,29}$ Chewing Alifix ${ }^{\circledR}$ the patient carries out a series of isometric contractions of the masticatory muscles and allows the development of a hypertrophy of the muscular fibers, which is accompanied by a better blood supply which allows the removal of the catabolites with a consequent reduction of the pain symptomatology and promotes moreover a greater irroration of oxygen, with consequent less tendency to hypoxia during muscular job. The effect of the increase in muscular volume is manifested by an increase in work capacity and therefore in muscle strength. It is hypothesized that the use of Alifix ${ }^{\circledR}$ can favor the conversion of IIA 


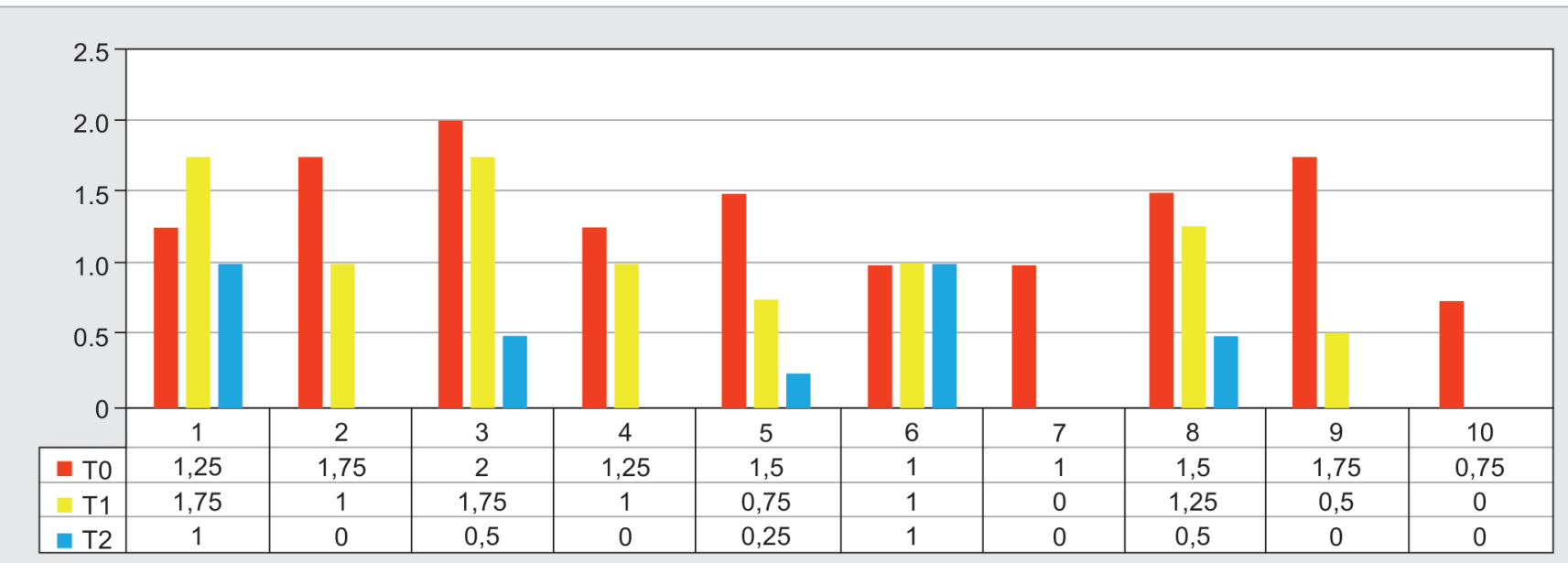

Fig. 6: Total mean pain from T0 (before treatment) to T2 (after 2 months of treatment with Alifix ${ }^{\circledast}$ )

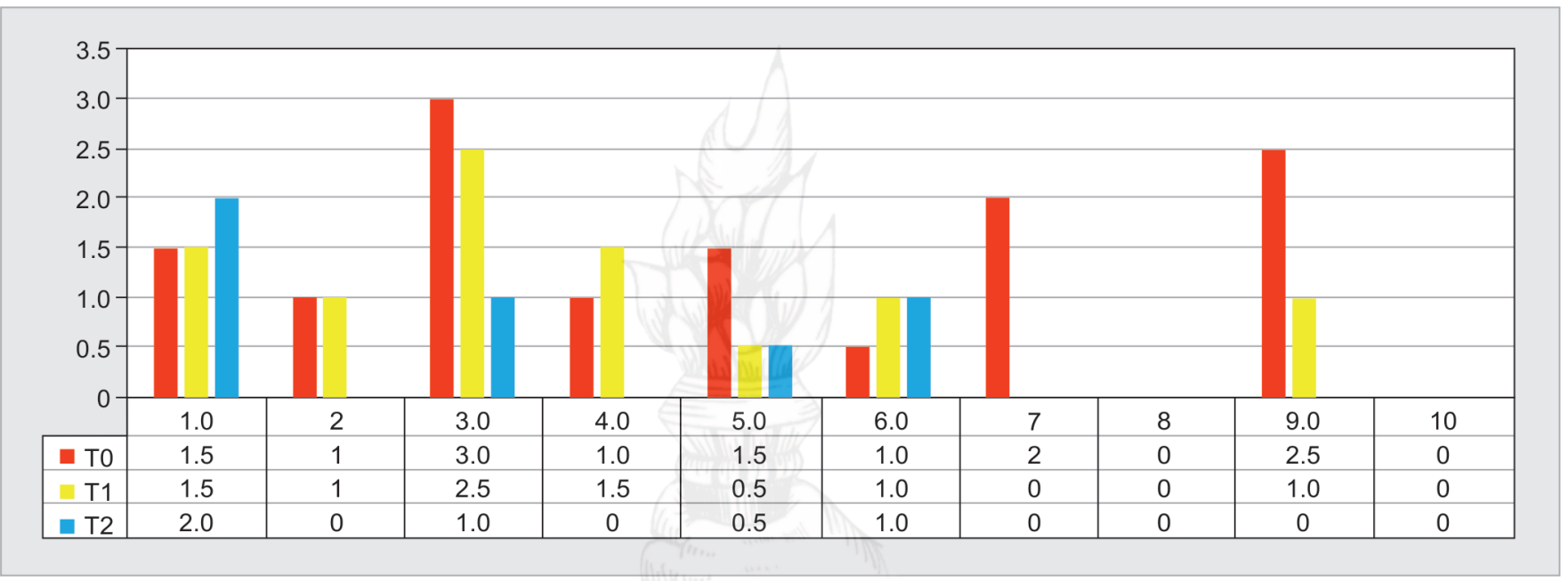

Fig. 7: Mean pain of temporal muscles from T0 (before treatment) to T2 (after 2 months of treatment with Alifix ${ }^{\oplus}$ )

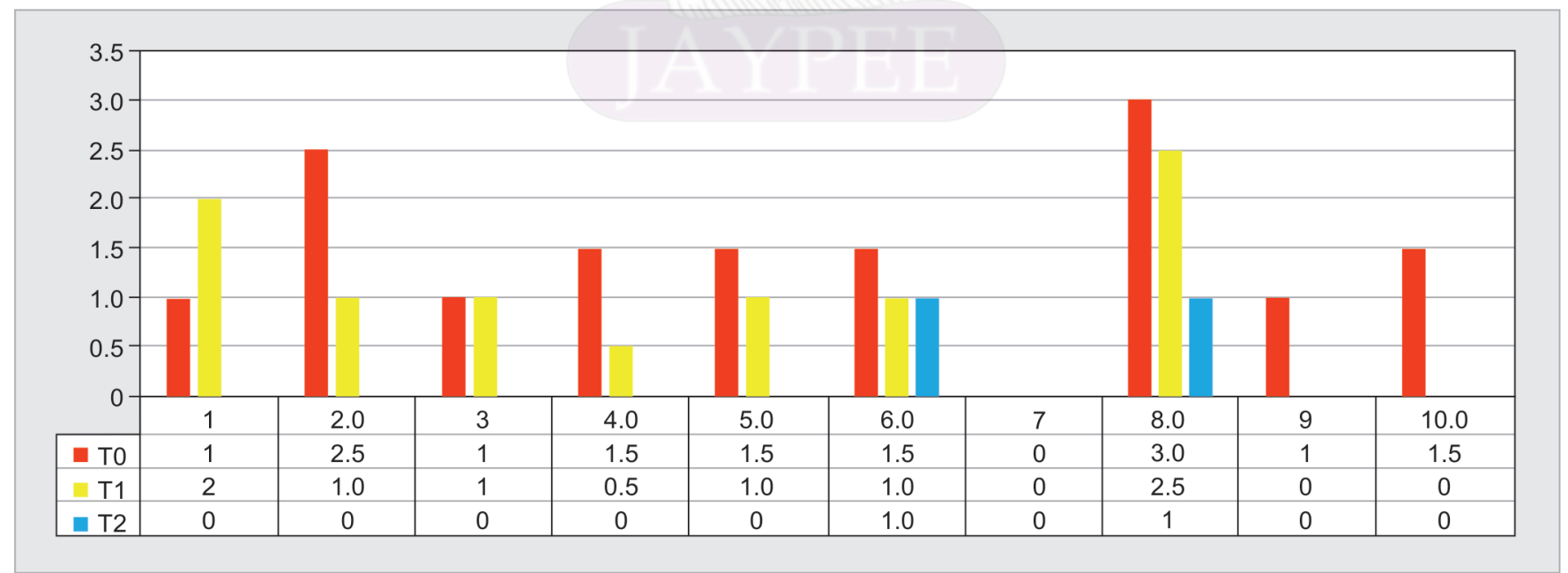

Fig. 8: Mean pain of masseters from T0 (before treatment) to T2 (after 2 months of treatment with Alifix ${ }^{\circledast}$ )

type muscle fibers into type I muscle fibers, with slow contraction and more resistant to neuromuscular fatigue. These considerations are based on the ability of skeletal muscles to change their anatomical, histochemical, and biochemical characteristics according to the stimuli to which they are subjected. Changes in the type and size of fibers are often associated with changes in intensity, duration, and frequency of muscle activation by the central nervous system. ${ }^{23,28,29}$ 


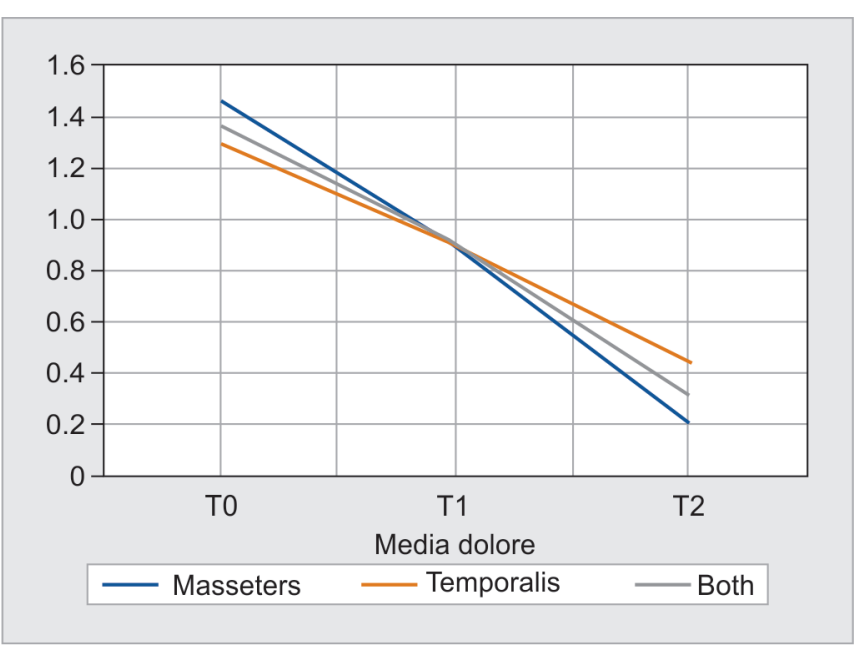

Fig. 9: Variations in total muscular pain

Typically, resistance training of a skeletal muscle, for example, by repeated isometric contraction, causes an increase in muscle thickness and improves muscle strength. Therefore, the section of the fibers increases, both slow and fast ones, with consequent hypertrophy. Moreover, fibers subjected to exercise form more myofilaments and myofibrils, store more glycogen, and have a greater mitochondrial content and a greater aerobic-oxidative potential of energy supply, which makes them more resistant to neuromuscular fatigue. It should be noted that adaptive changes in dimensions are completely reversible. ${ }^{30,31}$

From studies carried out in rats by Manabe et al., it has been seen that in the skeletal muscles subjected to training the glycogen content increased and that this phenomenon was accompanied by an increase in the expression of the glycogen synthase protein, the activity of glycogen synthase, and the activity of PP1 (protein phosphatase 1). ${ }^{32}$ It is assumed that these events also occur in the muscles of mastication following the use of Alifix ${ }^{\circledast}$.

Alifi $\mathrm{x}^{\circledast}$ during its use eliminates the dental contact in order to stop the occlusal input and muscular imbalance and achieve muscle relaxation. Thanks to the two separate parts, it moves independently and continuously during chewing. This offers another important advantage from the neurophysiological point of view: it determines a continuous variation of proprioceptive stimuli, increasing the wealth of information for muscle rebalancing. By asking the patient to place the horizontal part of Alifix ${ }^{\circledast}$ with the greater thickness placed anteriorly or posteriorly depending on its greater comfort, it is possible to compensate imbalances in the dental support. A greater posterior thickness compensates the lack of posterior support, activates more the masseter muscle, and promotes a cervical muscular organization that determines a straightening of the head. A greater anterior thickness, on the contrary, compensates for the lack of anterior support and activates more the temporal muscle, facilitating the extension of the head. ${ }^{33,34}$

\section{Clinical Significance}

Alifix ${ }^{\circledast}$ has proven to be a useful device for resolving the algic symptomatology in TMD of muscular origin. Muscle function, assessed by surface EMG, showed an improvement in the POC
TA, POC MM, BAR, and TORS indexes, even if not statistically significative. From these considerations, we conclude that the efficacy of Alifix ${ }^{\oplus}$ is clinically demonstrated.

However, further studies involving a larger sample and taking longer duration of therapy are needed. It is possible that the use of Alifix ${ }^{\circledR}$ over time may bring the EMG values within the normal ranges, bringing a statistically significant improvement on the degree of muscular balance.

This study presented as a limit the patient's collaboration in performing the recommended exercises, as $40 \%$ of patients revealed that they were not consistent with Alifix ${ }^{\circledast}$ therapy.

The worsening of the painful condition, the absence of benefits, or the decrease in pain possibly prompted the patients not to feel the need to perform the therapy consistently.

It is likely that patients who have reported pain increase have exaggerated with the chewing force during the exercises or have not stopped in case of signs of fatigue or tension as prescribed by the operator.

Another limitation is the subjective nature of pain, which cannot be measured by instrumental investigations but only described by patients. Finally, a further limit is the small number of the sample. It should be noted that three patients who received Alifix ${ }^{\circledR}$ did not show up at the controls and therefore were not included in the series.

\section{References}

1. Cairns BE. Pathophysiology of TMD pain-basic mechanisms and their implications for pharmacotherapy. J Oral Rehabil 2010;37(6):391-410. DOI: 10.1111/j.1365-2842.2010.02074.x.

2. Douglas CR, Avoglio JL, de Oliveira H. Stomatognathic adaptive motor syndrome is the correct diagnosis for temporomandibular disorders. Med Hypotheses 2010;74(4):710-718. DOI: 10.1016/ j.mehy.2009.10.028.

3. Ferrario VF, Sforza C, Tartaglia GM, et al. Immediate effect of a stabilization splint on masticatory muscle activity in temporomandibular disorder patients. J Oral Rehabil 2002;29(9): 810-815. DOI: 10.1046/j.1365-2842.2002.00927.x.

4. Ferrario VF, Tartaglia GM, Luraghi FE, et al. The use of surface electromyography as a tool in differentiating temporomandibular disorders from neck disorders. Man Ther 2007;12(4):372-379. DOI: 10.1016/j.math.2006.07.013.

5. Landulpho $A B$, E Silva $W A B$, E Silva $F A$, et al. Electromyographic evaluation of masseter and anterior temporalis muscles in patients with temporomandibular disorders following interocclusal appliance treatment. J Oral Rehabil 2004;31(2):95-98. DOI: 10.1046/j.0305182x.2003.01204.x.

6. Suvinen $\mathrm{TI}$, Reade PC, Kononen M, et al. Vertical jaw separation and masseter muscle electromyographic activity: a comparative study between asymptomatic controls and patients with temporomandibular pain and dysfunction. J Oral Rehabil 2003;30(8): 765-772. DOI: 10.1046/j.1365-2842.2003.01114.x.

7. DeVocht JW, Long CR, Zeitler DL, et al. Chiropractic treatment of temporomandibular disorders using the activator adjusting instrument: a prospective case series. J Manipulative Physiol Ther 2003;26(7):421-425. DOI: 10.1016/S0161-4754(03)00096-4.

8. Tartaglia GM, Moreira Rodrigues da Silva MA, Bottini S, et al. Masticatory muscle activity during maximum voluntary clench in different research diagnostic criteria for temporomandibular disorders (RDC/TMD) groups. Man Ther 2008;13(5):434-440. DOI: 10.1016/j.math.2007.05.011.

9. Roark AL, Glaros AG, O'Mahony AM. Effects of interocclusal appliances on EMG activity during parafunctional tooth contact. J Oral Rehabil 2003;30(6):573-577. DOI: 10.1046/j.1365-2842.2003.01139.x. 
10. Venino $\mathrm{PM}$, Citterio $\mathrm{CL}$, Pellegatta $\mathrm{A}$, et al. A micro-computed tomography evaluation of the shaping ability of two nickel-titanium instruments, hyflex EDM and protaper next. J Endod 2017;43(4): 628-632. DOI: 10.1016/j.joen.2016.11.022.

11. Maddalone $M$, Ferrari $M$, Stanizzi A, et al. Use of miniscrew implants in orthodontic distal movement. Dental Cadmos 2010;78(8):97-105.

12. Maddalone $M$, Ferrari $M$, Barrila' $S$, et al. Intrusive mechanics in orthodontics with the use of TAD's. Dental Cadmos 2010;78(7):97-106.

13. Kovalenko A, Slabkovskaya A, Drobysheva N, et al. The association between the psychological status and the severity of facial deformity in orthognathic patients. Angle Orthodontist 2012;82(3):396-402. DOI: 10.2319/060211-363.1.

14. Citterio F, Pellegatta A, Citterio CL, et al. Analysis of the apical constriction using micro-computed tomography and anatomical sections. Giornale Italiano di Endodonzia 2014;28:41-45.

15. Maddalone M, Gagliani M, Citterio $C L$, et al. Prevalence of vertical root fractures in teeth planned for apical surgery. A retrospective cohort study. Int Endod J 2018;51(9):969-974. DOI: 10.1111/iej.12910.

16. Khudanov BO, Abdullaev JR, Bottenberg $P$, et al. Evaluation of the fluoride releasing and recharging abilities of various fissure sealants. Oral Health Prev Dent 2018; 96-103. DOI: 10.3290/j.ohpd.a39823.

17. Caccianiga G, Paiusco A, Perillo L, et al. Does low-level laser therapy enhance the efficiency of orthodontic dental alignment? results from a randomized pilot study. Photomed Laser Surg 2017;35(8):421-426. DOI: 10.1089/pho.2016.4215.

18. Porcaro G, Busa A, Bianco E, et al. Use of a partial-thickness flap for guided bone regeneration in the upper jaw. J Contemp Dent Pract 2017;18(12):1117-1121. DOI: 10.5005/jp-journals-10024-2186.

19. Porcaro G, Tremolizzo L, Appollonio I, et al. Persistent hiccup reflex activation as a complication of dental implant surgery: a case report. Oxf Med Case Reports 2018;2018(6):199-201.

20. Galluzzi F, Pignataro L, Maddalone M, et al. Recurrences of surgery for antrochoanal polyps in children: a systematic review. Int J Pediatr Otorhinolaryngol 2018;106:26-30. DOI: 10.1016/j.ijporl.2017.12.035.

21. Ambu E, Citterio CL, Pellegatta $A$, et al. The use of limited CBCT in the early diagnosis of root vertical fracture: a case report. Glob J Oral Sci 2018;4:18-24.

22. Kogawa EM, Calderon PS, Lauris JRP, et al. Evaluation of maximal bite force in temporomandibular disorders patients. J Oral Rehabil 2006;33(8):559-565. DOI: 10.1111/j.1365-2842.2006.01619.x.

23. Kiliaridis S, Tzakis MG, Carlsson GE. Effects of fatigue and chewing training on maximal bite force and endurance. Am J Orthod
Dentofacial Orthop 1995;107(4):372-378. DOI: 10.1016/s08895406(95)70089-7.

24. Thompson DJ, Throckmorton GS, Buschang PH. The effects of isometric exercise on maximum voluntary bite forces and jaw muscle strength and endurance. J Oral Rehabil 2001;28(10):909-917. DOI: 10.1046/j.1365-2842.2001.00772.x.

25. Masumoto N, Yamaguchi K, Fujimoto S. Daily chewing gum exercise for stabilizing the vertical occlusion. J Oral Rehabil 2009;36(12): 857-863. DOI: 10.1111/j.1365-2842.2009.02010.x.

26. Schiffman E, Ohrbach R, Truelove E, et al. Diagnostic criteria for temporomandibular disorders (DC/TMD) for clinical and research applications: recommendations of the international RDC/TMD consortium network and orofacial pain special interest group. J Oral Facial Pain Headache 2014;28(1):6-27. DOI: 10.11607/jop.1151.

27. Ferrario VF, Tartaglia GM, Galletta A, et al. The influence of occlusion on jaw and neck muscle activity: a surface EMG study in healthy young adults. J Oral Rehabil 2006;33(5):341-348. DOI: 10.1111/j.13652842.2005.01558.x.

28. Catanzariti JF, Debuse T, Duquesnoy B. Chronic neck pain and masticatory dysfunction. Joint Bone Spine 2005;72(6):515-519. DOI: 10.1016/j.jbspin.2004.10.007.

29. Pimenta Ferreira $C L$, Bellistri G, De Felicio $C M$, et al. Patients with myogenic temporomandibular disorders have reduced oxygen extraction in the masseter muscle. Clin Oral Invest 2017;21(5): 1509-1518. DOI: 10.1007/s00784-016-1912-2.

30. Grünheid T, Langenbach GEJ, Korfage JAM, et al. The adaptive response of jaw muscles to varying functional demands. Eur J Orthod 2009;31(6):596-612. DOI: 10.1093/ejo/cjp093.

31. Davis NW. Modulation of ATP-sensitive $\mathrm{K}+$ channel in skeletal muscle by intracellular protons. Nature 1990;343(6256):375-377. DOI: 10.1038/343375a0.

32. Manabe Y, Gollisch KSC, Holton L, et al. Exercise traininginduced adaptations associated with increases in skeletal muscle glycogen content. FEBS J 2013;280(3):916-926. DOI: 10.1111/febs. 12085.

33. Carini F, Longoni S, Pisapia V, et al. Implant-supported prostheses with temporomandibular joint reproduction after hemimandibular resection: a case report. Ann Stomatol (Roma) 2014;5(Suppl 2 to No 2):1-9.

34. Hruby RJ. The total body approach to the osteopathic management of temporomandibular joint dysfunction. J Am Osteopath Assoc 1985;85(8):502-510. 